\title{
An acid-sensing ion channel that detects ischemic pain
}

L.A. Naves ${ }^{1}$ and E.W. McCleskey ${ }^{2}$
1Departamento de Fisiologia e Biofísica, Instituto de Ciências Biológicas, Universidade Federal de Minas Gerais, Belo Horizonte, MG, Brasil

${ }^{2}$ Vollum Institute, Oregon Health Science University, Portland, OR, USA

\footnotetext{
Correspondence

L.A. Naves

Departamento de Fisiologia e

Biofísica, ICB-UFMG

Avenida Antônio Carlos, 6627

31270-901 Belo Horizonte, MG

Brasil

Fax: +55-31-3499-2924

E-mail Inaves@icb.ufmg.br

Presented at the

XIX Annual Meeting of the

Federação de Sociedades de

Biologia Experimental,

Águas de Lindóia, SP, Brazil,

August 25-29, 2004.

$\ldots \ldots \ldots \ldots \ldots \ldots \ldots$
}

Received February 23, 2005

Accepted May 19, 2005

\begin{abstract}
Ischemic pain occurs when there is insufficient blood flow for the metabolic needs of an organ. The pain of a heart attack is the prototypical example. Multiple compounds released from ischemic muscle likely contribute to this pain by acting on sensory neurons that innervate muscle. One such compound is lactic acid. Here, we show that ASIC3 (acid-sensing ion channel \#3) has the appropriate expression pattern and physical properties to be the detector of this lactic acid. In rats, it is expressed only in sensory neurons and then only on a minority $(\sim 40 \%)$ of these. Nevertheless, it is expressed at extremely high levels on virtually all dorsal root ganglion sensory neurons that innervate the heart. It is extraordinarily sensitive to protons (Hill slope 4 , half-activating $\mathrm{pH}$ 6.7), allowing it to readily respond to the small changes in extracellular $\mathrm{pH}$ (from 7.4 to 7.0 ) that occur during muscle ischemia. Moreover, both extracellular lactate and extracellular ATP increase the sensitivity of ASIC3 to protons. This final property makes ASIC3 a "coincidence detector" of three molecules that appear during ischemia, thereby allowing it to better detect acidosis caused by ischemia than other forms of systemic acidosis such as hypercapnia.
\end{abstract}

Key words

- Pain

- Ischemia

- Dorsal root ganglia

- Acid-sensing ion channels

- Lactic acidosis

...............

\section{Ischemic pain}

Three diseases are characterized by ischemic pain: angina, intermittent claudication, and sickle cell anemia. Angina is the chest pain caused by coronary vascular disease and intermittent claudication the pain caused by peripheral vascular disease. Misshapen red blood cells can block blood flow in sickle cell anemia, causing severe, unpredictable bouts of pain. Ischemic pain feels distinctly different from other forms of pain such as tissue damage, burning, or inflammation. The sensation can be experienced simply by running or swimming at a sprint for about 40 or $50 \mathrm{~s}$. The severity of the muscle pain will stop you from proceeding further. It is this sensation that appears unexpectedly in sickle cell or angina patients, or upon mild exercise, such as walking, in a patient with intermittent claudication. Middle distance athletes and thoroughbred racehorses train to maximize the time they can sprint before being stopped by muscle ischemia.

The most important fact about ischemic pain was demonstrated in experiments performed in the 1930's. Blocking blood flow to an arm with a tourniquet does not, of itself, cause pain. But if it is accompanied by mild forearm exercise, striking ischemic pain ensues (1). The conclusion is that the pain is caused by release of one or more compounds 
from working muscle that accumulate when there is insufficient blood flow and then act upon certain nociceptive sensory neurons that innervate the muscle. Much of the ensuing effort in the field has asked what the mediating compounds and their receptors on sensory neurons are. Table 1 lists the most widely considered compounds that are reported to cause pain when perfused into the hearts of intact animals (2-13).

\section{The lactic acid paradoxes}

Lactic acid is produced by anaerobic metabolism when muscle gets insufficient oxygen. This and the fact that acid is commonly considered a painful stimulus suggest that lactic acid mediates ischemic pain. But two experimental facts argue otherwise. First, extracellular $\mathrm{pH}$ drops to about neutral during ischemic pain, but not much further $(14,15)$. Neutral $\mathrm{pH}$ solutions surely do not cause pain when poured onto a skinned knee, so the field has been reluctant to believe that such a modest $\mathrm{pH}$ change could be the source of ischemic pain. The second fact is that similar $\mathrm{pH}$ changes can occur during metabolic problems involving the lungs or kidney. However, metabolic acidosis is not accompanied by angina-like chest pain.

Nevertheless, a compelling experiment by Pan et al. (13) argues that $\mathrm{pH}$ must play a role. These investigators measured the electrical activity of sensory nerve fibers coming

Table 1. Putative mediators of ischemic pain.

\begin{tabular}{ll}
\hline Mediators & References \\
\hline $\begin{array}{l}\text { Bradykinin } \\
\text { Serotonin }\end{array}$ & Baker et al. (2); Tjen-A-Looi et al. (3) \\
Jdenosine & Games et al. (4) \\
Non-adenosine & Abe et al. (7); Pan and Longhurst (8) \\
Adenosine 5'-triphosphate & Huang et al. (9) \\
Substance P & Huang et al. (9) \\
Oxygen radicals & Huang et al. (10) \\
Histamine & Ginsburg et al. (11) \\
Protons & Neill (12); Pan et al. (13) \\
Lactate & Neill (12); Pan et al. (13) \\
\hline
\end{tabular}

from the heart and then blocked blood flow to a coronary artery. The resulting increase in nerve firing was greatly diminished if a high concentration of a $\mathrm{pH}$ buffer was present when the artery was blocked. Evidently, the buffer intercepted the protons before they reached their receptor on the sensory axon and thereby diminished the resulting pain signal. But, what receptor works to detect the small change in $\mathrm{pH}$ and how can it distinguish between a $\mathrm{pH}$ change that is caused by ischemia from one caused by systemic metabolic problems?

\section{Acid-sensing ion channels}

The results presented in this paper will demonstrate that acid-sensing ion channel number 3 (ASIC3) has the appropriate physical properties and expression pattern to selectively sense the acid that accompanies ischemia. ASIC were discovered in the early 1980's by Oleg Krishtal and his colleagues in Kiev (16-18). These investigators demonstrated that acid triggered the opening of ion channels in sensory neurons and showed that there were several kinetically distinct acid-sensing channels expressed on different sensory neurons. Krishtal demonstrated the basic properties of the channels: gating by acid, sodium-selective pores, and weak block by amiloride. Further work also showed the presence of ASIC on neurons of the central nervous system $(19,20)$.

Largely dormant for a decade after Krishtal's work (21), the field was resurrected in 1997 when Michel Lazdunski's laboratory cloned an acid-sensing channel and demonstrated that it is a member of a large family of channels that have been extensively studied. The family is commonly called the degenerins/epithelial sodium channels $(\mathrm{ENaC})$ (18) and is characterized by amiloride sensitivity and sodium selectivity (Figure 1). These channels have no homology to voltage-gated sodium channels but, instead, have about 500 amino acids, two 
putative transmembrane domains, intracellular $\mathrm{N}$ and $\mathrm{C}$ terminals, and a substantial extracellular domain. At least three subunits must bind together to form a functional channel. The most studied members of this family are the ENaCs. ENaCs are constitutively active, sodium selective channels that are expressed on epithelia and support selective sodium transport across them. Degenerin channels are expressed in C. elegans worms. A mutation that causes them to be overactive causes degeneration of mechanosensing neurons, this being one reason why they are considered to be mechanosensing channels in worms.

Extensive cloning work has found four distinct genes with appropriate homology to be considered in the ASIC family (22). There are also two known splice variants of ASIC1 and two of ASIC2. ASIC4 forms no functional channel and it takes fairly extreme acid (pH 5 and below) to open ASIC2. But ASIC 1 and ASIC 3 are opened by $\mathrm{pH}$ changes that are clearly relevant physiologically. ASIC3 is expressed predominately in the sensory peripheral nervous system and is not seen in the central nervous system. ASIC1 is the predominant ASIC in the central nervous system and is also present in sensory neurons. ASIC2 is present both in the central and peripheral nervous systems and forms heteromultimers with ASIC1 and ASIC3, causing detectable changes in either kinetics or pharmacology.

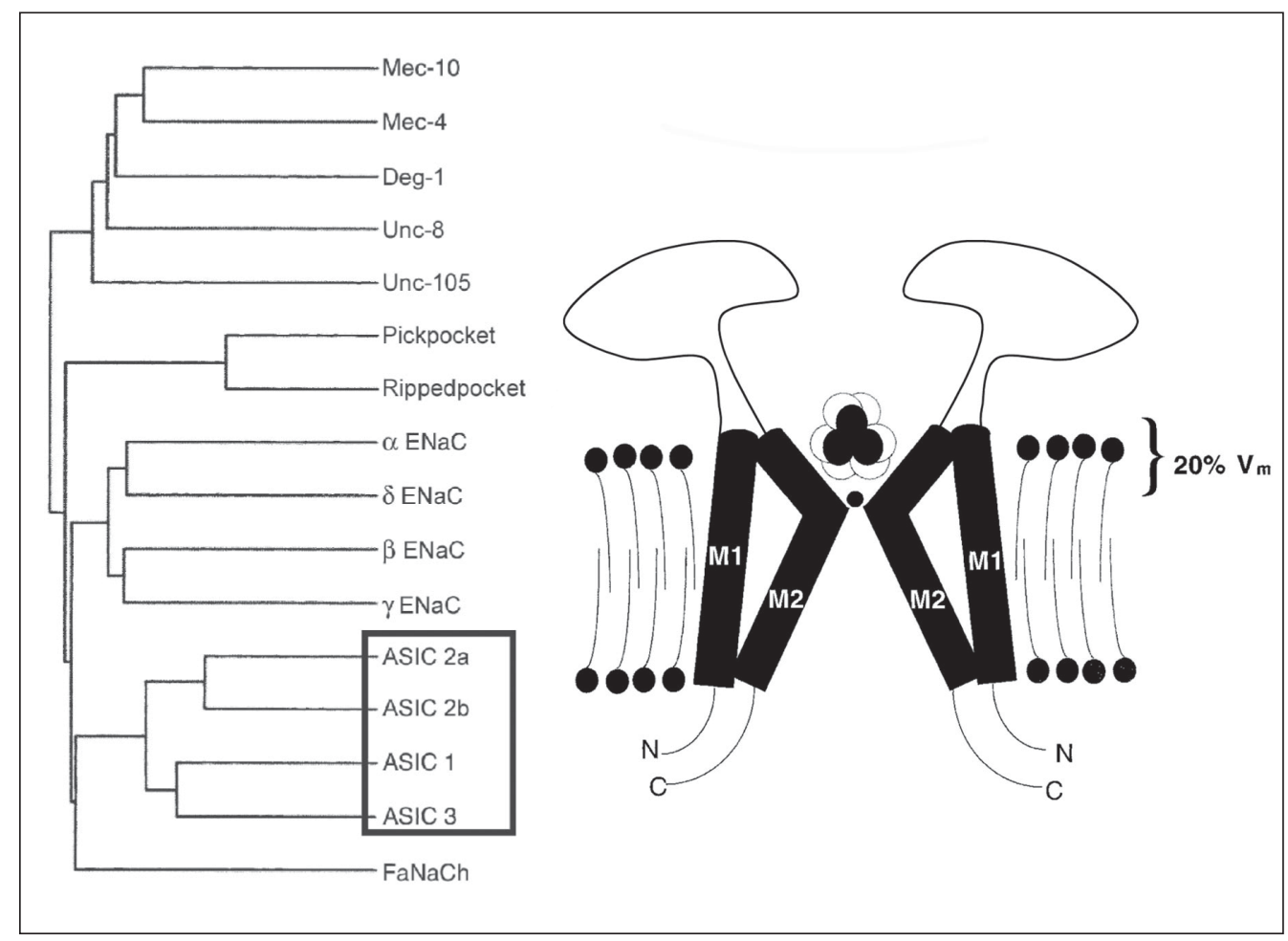

Figure 1. Amiloride-sensitive sodium channels. The homology tree on the left shows the relationship of acidsensing ion channels (ASICs) to other members of the epithelial sodium channels and degenerins (ENaC-Deg) family. Besides the degenerins from C. elegans (Mec-10, Mec-4, Unc-8, and Unc-105), the family includes Drosophila channels (Pickpocket and Rippedpocked), vertebrate channels (ENaCs and ASICs) and the FMRFamide peptide-gated sodium channel ( $\mathrm{FaNaCh}$ ) expressed in Helix aspersa. The proposed model for ENaCs on the right shows two channel subunits, each composed of two transmembrane domains (M1 and M2). The small black circles represent a dehydrated sodium or lithium ion near the selectivity filter, and the guanidinium group of amiloride, which is able to penetrate $20 \%$ of the electrical field, is also shown. $\mathrm{N}$ and $\mathrm{C}=$ intracellular $\mathrm{N}$ and $\mathrm{C}$ terminals. Adapted, with permission, from Annual Review of Physiology, 62: 573, 2000 [www.annualreviews.org]. 


\section{A method to isolate ischemia-sensing neurons}

Our contributions to the study of ischemic pain use the single-cell patch clamp method applied to dissociated sensory neurons. The most significant disadvantage of this approach compared to the traditional whole animal preparations is that one cannot readily distinguish the sensory modality of individual neurons once they have been dissociated from the animal. We addressed this problem with an in vivo retrograde labeling strategy (Figure 2).

The rationale of the method takes advan- tage of the fact that the heart is a most unusual sensory organ. Pain is the only conscious sensation that ever arises from the heart muscle and the only thing that can cause the pain is ischemia. Cutting or burning heart muscle causes no pain, but blocking a blood vessel does. There may also be subconscious sensory signals involved in cardiovascular reflexes. But it can be said with certainty that most, and perhaps all, sensory neurons that innervate heart muscle are specialized to detect ischemia. Thus, if we could label them, we would have labeled ischemia sensors.

We injected into the pericardial space a
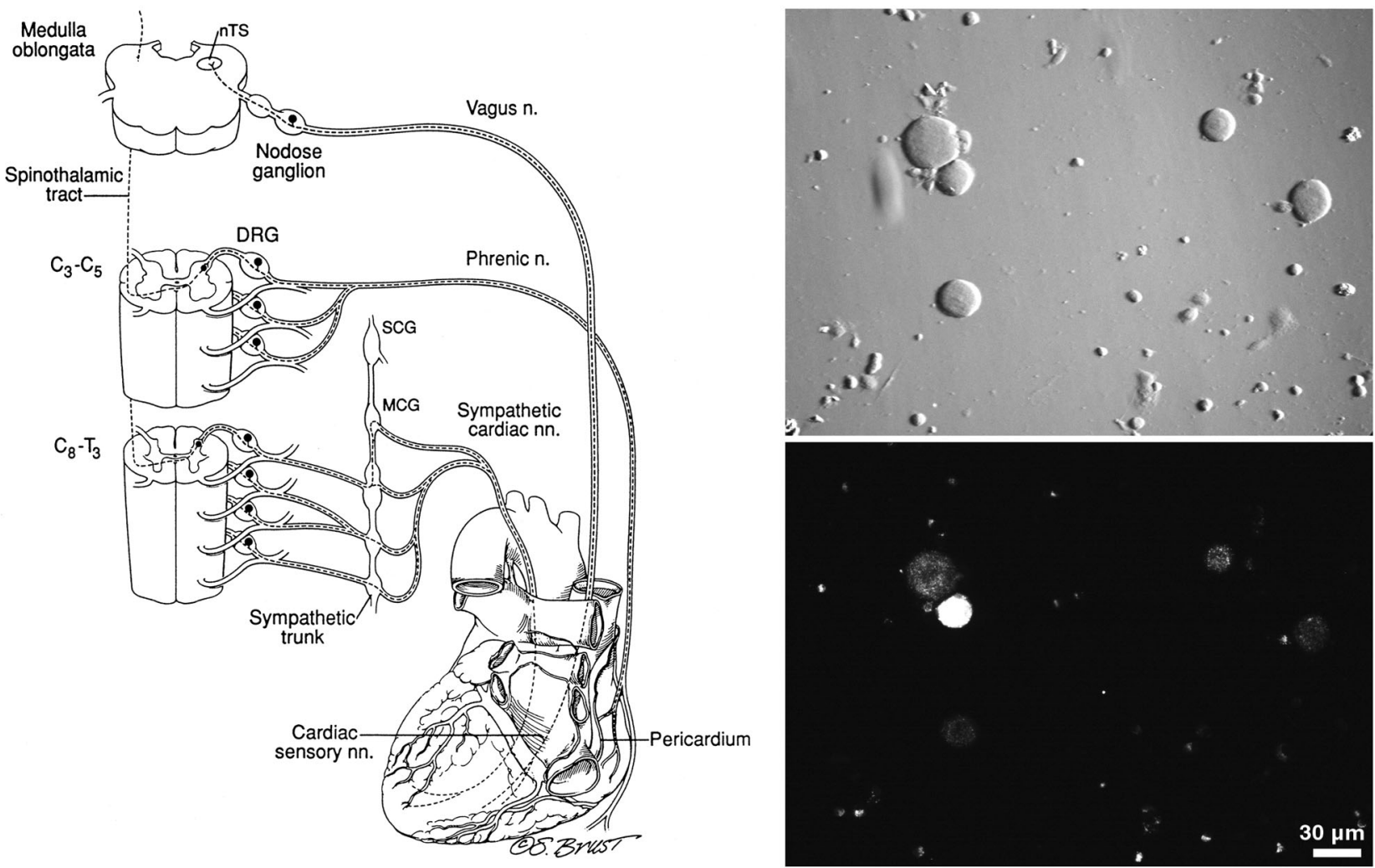

Figure 2. Retrograde labeling identifies dissociated neurons which innervate heart muscle. Left side: cardiac sensory neurons innervating the heart can be found in the lower cervical to upper thoracic $\left(\mathrm{C}_{8}-\mathrm{T}_{3}\right)$ dorsal root ganglia (DRG) and in the nodose ganglia, while afferent neurons from the pericardium have their cell bodies in the DRG of the upper cervical region $\left(\mathrm{C}_{3}-\mathrm{C}_{5}\right)$. Reproduced, with permission, from Benson et al. (23), American Heart Association. Right side: brightfield (upper) and fluorescent (bottom) photos of dissociated $\mathrm{C}_{8}-\mathrm{T}_{3} \mathrm{DRG}$ neurons. Faint background fluorescence is present in four of the five sensory neurons, whereas one is brightly fluorescent from retrogradely transported dye (Dil) injected into the pericardial space. $\mathrm{nTS}$ = nucleus tractus solitarius; SCG and MCG = superior and middle cervical ganglion, respectively. Reproduced with permission, from Immke and McCleskey (24), Nature Publishing Group [www.nature.com/neuro/]. 
lipid-soluble dye called 1,1'-dioctadecyl3,3,3',3'-tetramethylindocarbocyanine perchlorate (DiI). DiI intercalates into lipid membranes at the site of injection, including local nerve endings. The resulting fluorescent membrane gets endocytosed and the intracellular fluorescent vesicles then use axoplasmic transport to travel to the cell body, located just outside the spinal cord at the level of the upper thoracic dorsal root ganglia. Therefore, when we dissect and dissociate dorsal root ganglia several weeks after injecting the dye, we find some fluorescent neurons that must have innervated the heart muscle. We took these to be specialized ischemia sensors (23).

\section{Ischemia sensors express high levels of ASIC3}

Do ischemia-sensing neurons have properties that are different from those of other types of sensory neurons? We examined their array of ion channels using patch clamp methods. The most remarkable property is that they all have extraordinarily high currents evoked by decreasing $\mathrm{pH}$ (Figure 3). These currents dwarf those caused by other pain-causing agents, they are present in virtually all of the labeled neurons, and they are relatively rare in unlabeled neurons (23). This implies that the acid-triggered ion channel is important for detecting ischemia.

Which of the various ASICs mediate the current on the cardiac sensory neurons? To answer this, we expressed cDNA for each of the ASICs in COS7 cells and compared the functional properties of these known clones to the functional properties of the native channels in the cardiac sensory neurons (25). ASIC3 and the native channel were identical in all respects, whereas all other ASICs varied dramatically from the native channel. The most significant properties are: 1) the extremely steep activation curve (Hill slope $=4$; half-activation $\mathrm{pH} 6.7) ; 2$ ) $\mathrm{pH}$ at which half of the channels are desensitized $(\mathrm{pH}$
$7.2)$; 3) the rate of recovery from desensitization (ASIC3 and the native current recover quickly (less than $5 \mathrm{~s}$ ), whereas other ASICs take about $20 \mathrm{~s}$ ); 4) calcium permeability and block. We conclude that the bulk of the acid-triggered current in cardiac sensory neurons is carried through ion channels that include ASIC3. We have not yet deter-

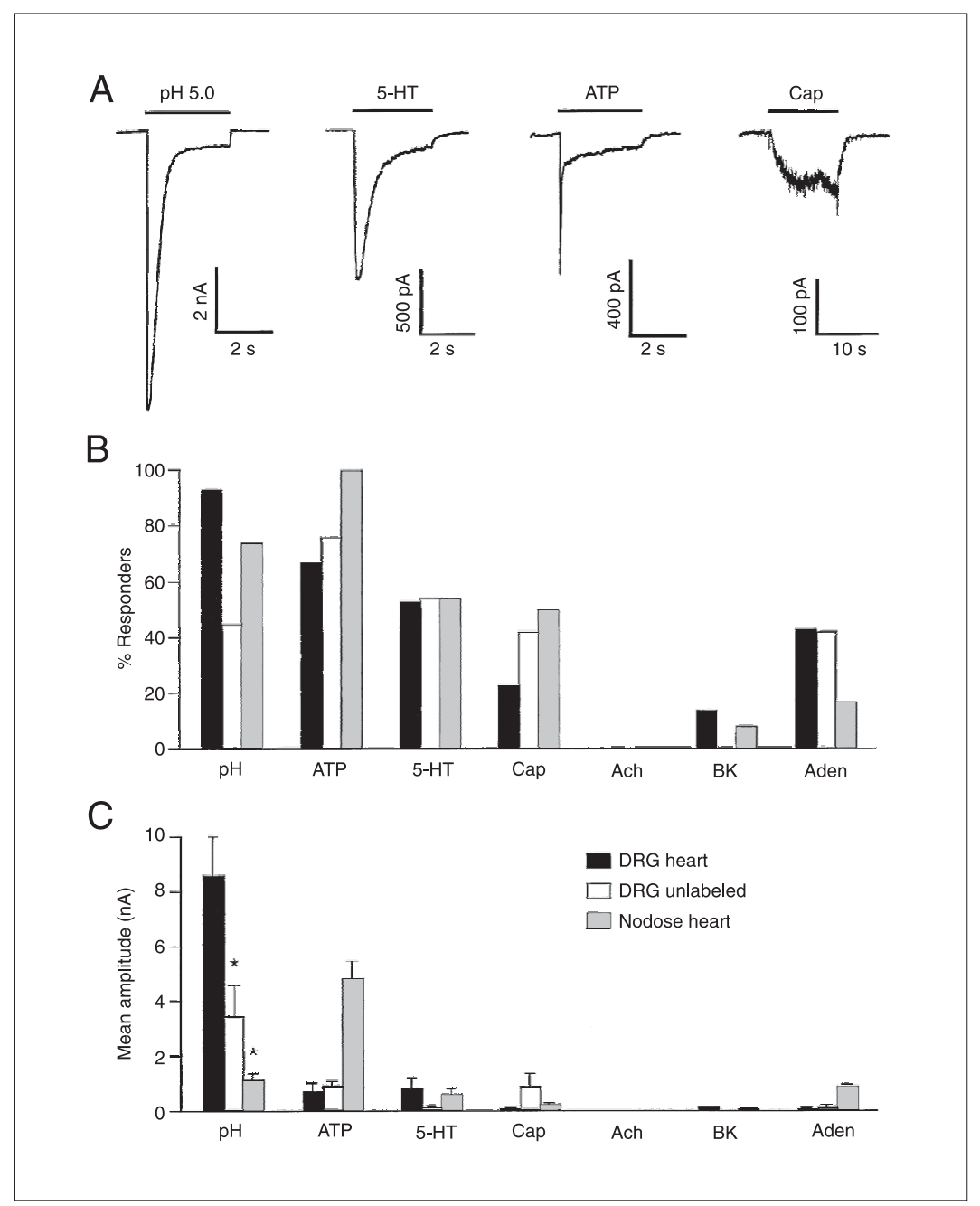

Figure 3. High levels of acid-sensing ion channel expression in cardiac sensory neurons. $A$, Representative currents evoked by application of the acid ( $\mathrm{pH} 5.0$ ), 5-hydroxytryptamine (5$\mathrm{HT}$ ), adenosine 5'-triphosphate (ATP) or capsaisin (Cap) to cardiac dorsal root ganglion (DRG) neurons. $B$, Percentage of labeled cardiac DRG neurons, unlabeled DRG neurons and labeled cardiac nodose neurons that responded to the following stimuli: $\mathrm{pH} 5.0,30 \mu \mathrm{M}$ ATP, $30 \mu \mathrm{M} 5-\mathrm{HT}, 1 \mu \mathrm{M}$ Cap, $200 \mu \mathrm{M}$ Aden, and $500 \mathrm{nM}$ BK. A positive response was defined as an evoked current $>50 \mathrm{pA}$. Each bar indicates the results for at least 12 cells. $C$, Mean $( \pm$ SEM) current amplitudes of the responding neurons. Ach $=$ acetylcholine, $B K=$ bradykinin, Aden $=$ adenosine. ${ }^{*} \mathrm{P}<0.01 \mathrm{vs} \mathrm{pH}$ evoked current in labeled cardiac DRG neurons (unpaired $t$-test). Reproduced, with permission, from Benson et al. (23), American Heart Association. 
mined if these channels are homomers of ASIC 3 or heteromers formed between ASIC3 and other ASICs.

\section{Paradox number 1 answered: extreme acid sensitivity of ASIC3}

Recall the lactic acid paradoxes mentioned above. The first was that the extracel-

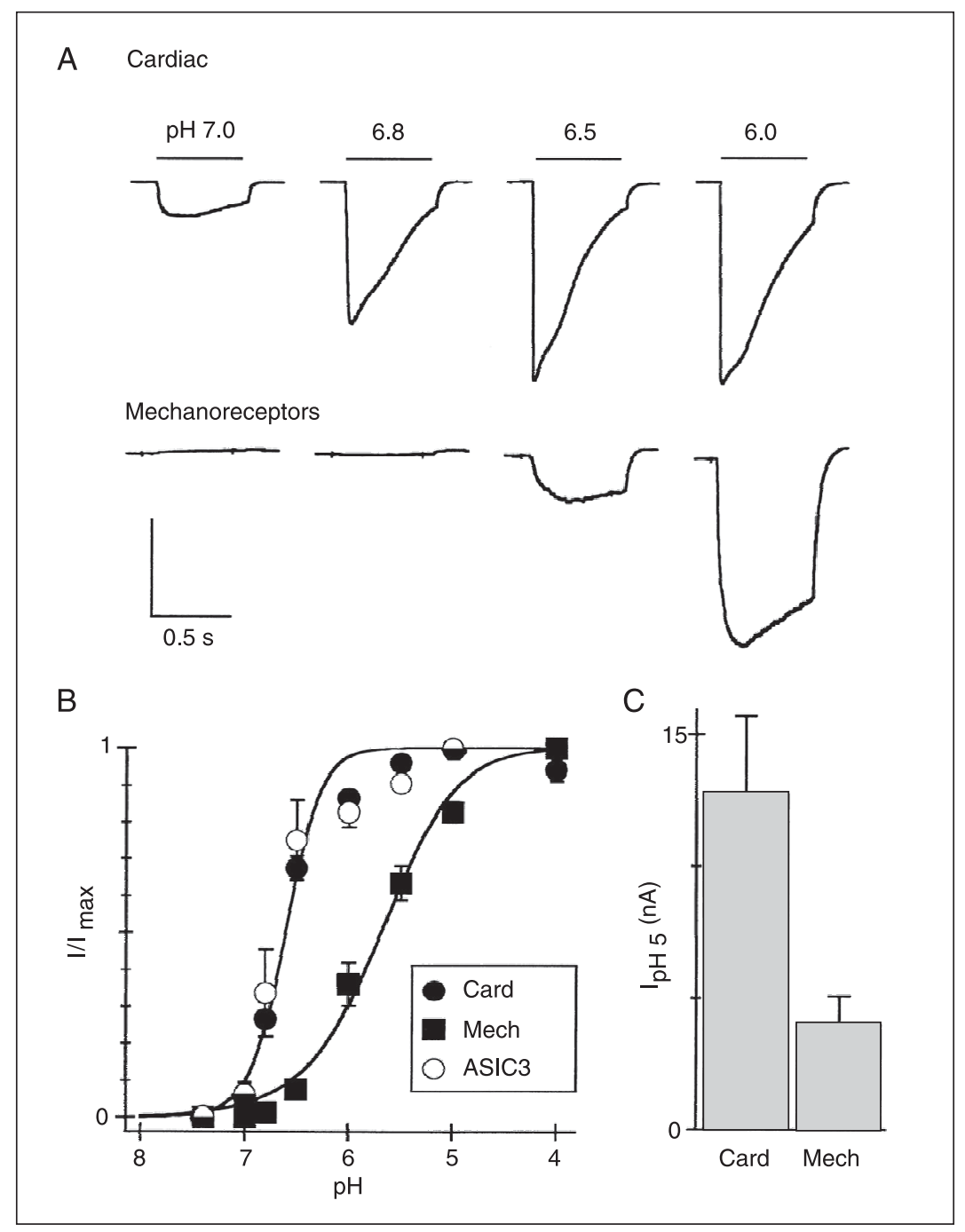

Figure 4. Labeled cardiac dorsal root ganglion neurons have highly sensitive acid-sensing ion channels (ASICs) which are similar to ASIC3 and distinct from those expressed by mechanoreceptors from the mesencephalic nucleus of the trigeminal nerve. $A$, Currents evoked from a cardiac afferent or a mechano-sensing afferent by pulses to the indicated $\mathrm{pH}$ from $\mathrm{pH}$ 8.0. Vertical scales: $8 \mathrm{nA}$ (upper), $1 \mathrm{nA}$ (lower). $B$, Mean ( $\pm \mathrm{SEM}$ ) fractional current vs $\mathrm{pH}$ for cardiac cells (Card), mechanoreceptors (Mech) and cells expressing ASIC3. $C$, Mean ( \pm SEM) amplitude of currents evoked by pH 5. Reproduced, with permission, from Sutherland et al. (25), National Academy of Sciences of the United States. lular $\mathrm{pH}$ changes that occur during ischemia are so small $(1 / 2 \mathrm{pH}$ unit) that people have doubted that this could be sufficient to trigger pain. ASIC3 answers this issue because it has extraordinarily high $\mathrm{pH}$ sensitivity in the range of extracellular $\mathrm{pH}$ that occurs during a heart attack (25). Figure 4 shows three activation curves. The ones for ASIC3 and the channel native to cardiac sensory neurons exactly overlap; another for a different kind of sensory neuron is far less sensitive to $\mathrm{pH}$. Figure 5 shows a higher resolution view of the $\mathrm{pH}$ range, 7.1 to 6.7 , that can occur during heart attack. ASIC3 reaches about $50 \%$ activation over this range, whereas other cloned ASICs are not so sensitive. The Hill coefficient for ASIC3 is about 4; this more or less means that ASIC3 is four times as sensitive as a $\mathrm{pH}$ meter over this critical range of $\mathrm{pH}$. Thus, we appear to have an answer to paradox number 1: ischemia-sensing neurons express high levels of ASIC3, which readily respond to the small $\mathrm{pH}$ changes that accompany a heart attack.

\section{Paradox number 2 answered:} coincident detection of lactate, ATP and acid

We are left with a seemingly more profound paradox: how can acid be relevant to ischemic pain if no pain is caused by metabolic events such as hypercapnia that can cause the same kind of $\mathrm{pH}$ change that occurs during a heart attack? Pan et al. (13) demonstrated the paradox most convincingly. They measured the $\mathrm{pH}$ on the surface of the heart when a coronary artery was blocked and found that it dropped from $\mathrm{pH} 7.4$ to 7.0. Then they reperfused the artery and had the animal breathe carbon dioxide until the resulting hypercapnia dropped the $\mathrm{pH}$ of the heart to 7.0. The blockade of the artery caused increased firing of sensory axons that innervate the heart, but the hypercapnia did not. How can this observation be reconciled with their other result (see above) that buffering 
extracellular $\mathrm{pH}$ greatly diminishes axon firing during artery occlusion? The simple interpretation is that protons must be necessary to activate the sensory axons, but cannot by themselves be sufficient. In other words, something must act together with protons to activate the axons.

We searched for compounds released during ischemia that might act together with protons to activate ASIC3. We found two: lactate and adenosine 5'-triphosphate (ATP). When the channel is activated by $\mathrm{pH} 7.0$ in the presence of $15 \mathrm{mM}$ lactate, the resulting current is $80 \%$ greater than when lactate is absent (Figure 6). These are physiological values. Under resting conditions, extracellular lactate is about $1 \mathrm{mM}$ in skeletal muscle; after extreme ischemic exercise it rises to 15-30 mM (26). The increased current in the presence of lactate makes the channel better at sensing the lactic acidosis that occurs in ischemia than other kinds of acidosis such as the carbonic acidosis when an animal breathes $\mathrm{CO}_{2}$.

Extracellular ATP rises to $>10 \mu \mathrm{M}$ when a muscle contracts without blood flow (27). We find that a transient appearance of such extracellular ATP can greatly increase ASIC3 current even for minutes after the ATP is removed (Figure 7 ).

Though they both increase ASIC3 current, lactate and ATP have qualitatively different effects. Lactate acts immediately and must be present for the ASIC current to be enhanced. ATP increases the current slowly - a peak is reached between $15 \mathrm{~s}$ and $1 \mathrm{~min}$ after ATP is applied - and the effect persists for minutes after ATP is removed. Also, lactate acts on every cell that expresses

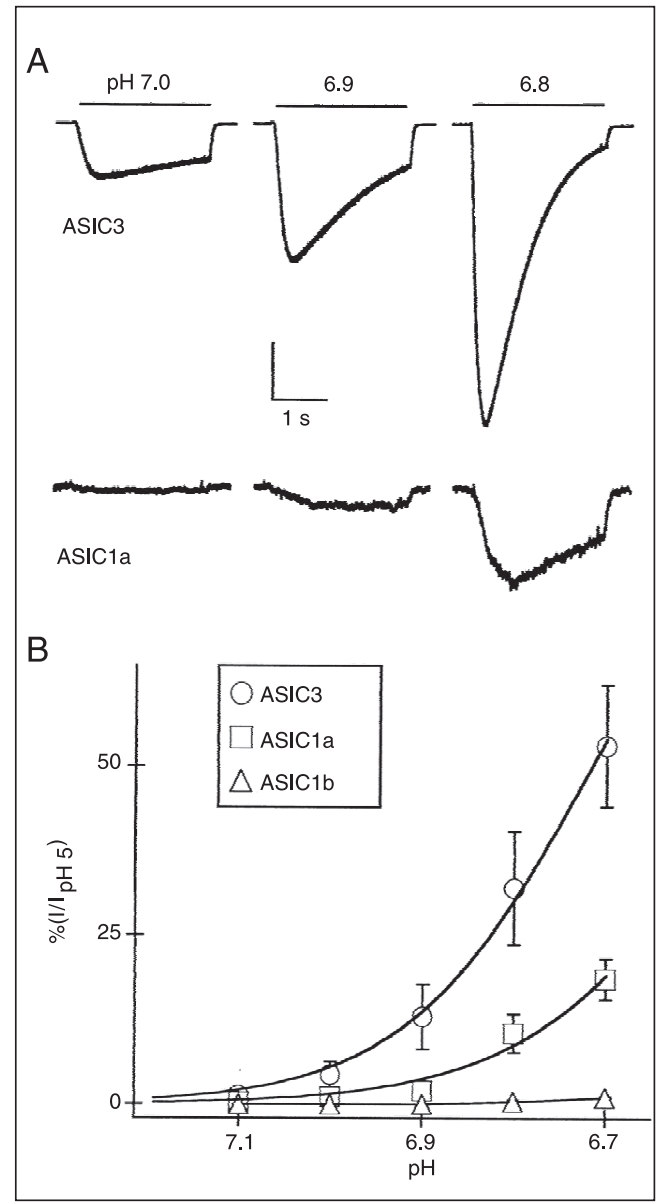

Figure 5. Acid-sensing ion channel 3 (ASIC3) opens at ischemic $\mathrm{pH}$. A, Currents evoked from cells expressing either ASIC3 or ASIC1a by steps to $\mathrm{pH} 7.0,6.9$, and 6.8. The vertical scale bar represents $10 \%$ of the current evoked by pH 5 (2 nA ASIC3; 46 pA ASIC1a). B, Average currents of the indicated clones normalized to the value at $\mathrm{pH} 5.0$ and fitted with the Hill equation. Hill coefficients are 4.3 (ASIC3, $\mathrm{pH}_{0.5}$ 6.7) and 3.9 (ASIC1a, $\mathrm{pH}_{0.5}$ 6.4). Reproduced, with permission, from Sutherland et al. (25), National Academy of Sciences of the United States.

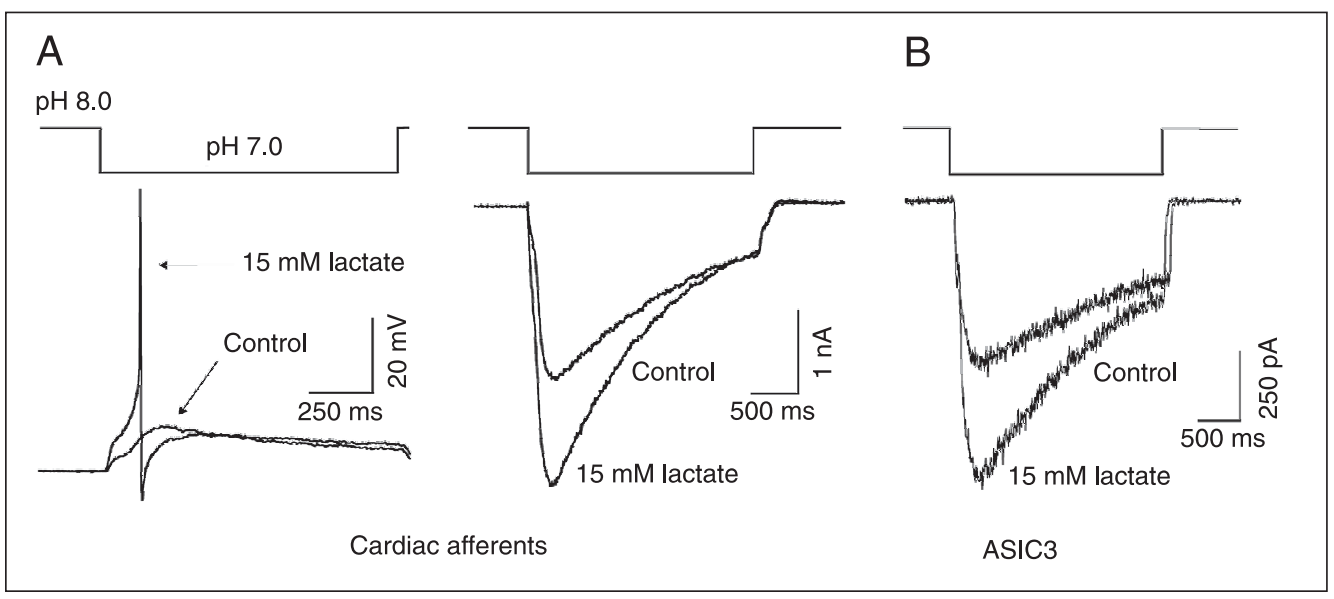

Figure 6. Lactate enhances the activity of acid-sensing ion channels (ASICs). A, Representative voltage (left) and current (right) recordings from a cardiac afferent dorsal root ganglion neuron briefly exposed to $\mathrm{pH} 7.0$ (resting $\mathrm{pH}=8.0$ ) in the presence or absence of $15 \mathrm{mM}$ lactate. $B$, Representative current recordings from a cell expressing ASIC3 using the same protocol as in A. Reproduced, with permission, from Immke and McCleskey (24), Nature Publishing Group [www.nature.com/neuro/]. 
Figure 7. Slow and persistent adenosine 5'-triphosphate (ATP) modulation of ASIC3-like currents in dorsal root ganglion neurons. A, Representative current recordings with changing external $\mathrm{pH}$ from 7.4 to 6.8 , before (control) and after $50 \mu \mathrm{M}$ ATP application. B, Time course of the ATP effect in one cell. Current sizes before, during and after ATP application for $70 \mathrm{~s}$. A second ATP application did not enhance the acid current to the same extent. From Fierro LP, Naves LA, Spelta $V$ and McCleskey EW (unpublished data)

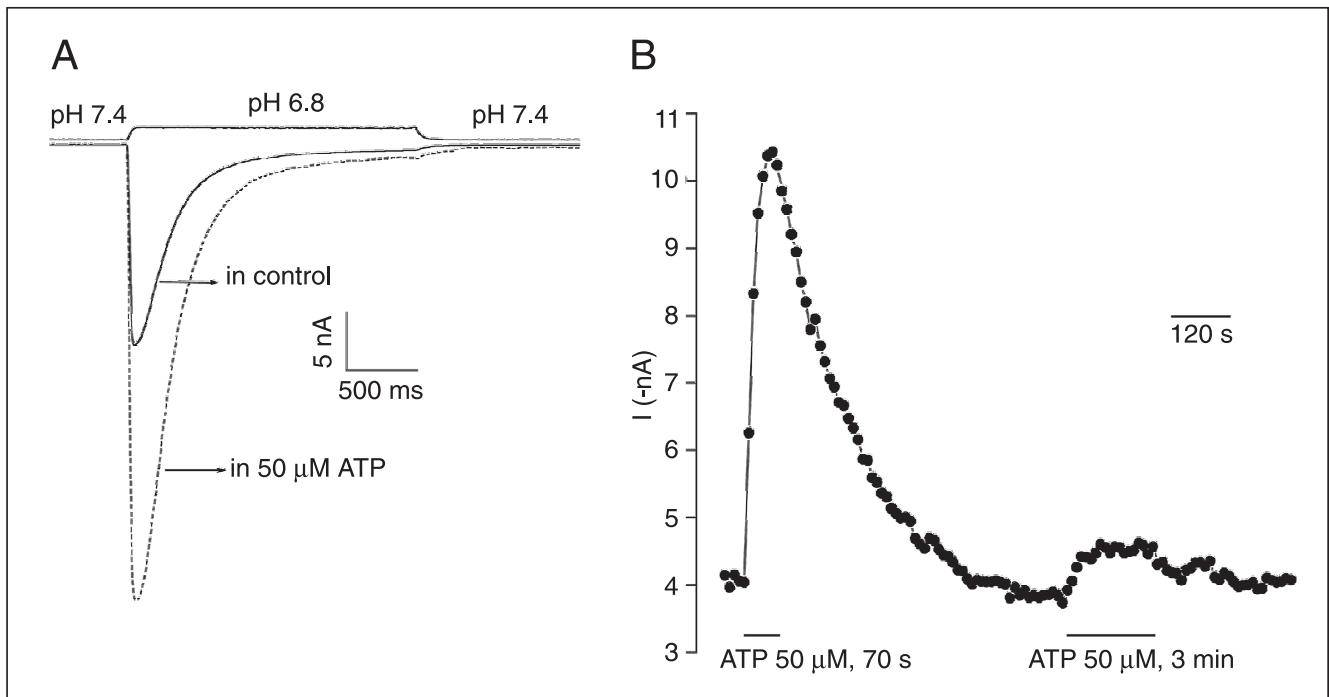

ASIC3 whereas ATP acts on some cells but not others. We find that lactate acts by altering the basic gating of the channel, which, surprisingly, involves binding of calcium in addition to protons (28). In contrast, the ATP binding site must not be the ASIC3 channel itself; there are a variety of purinergic receptors, some of which are ion channels and some of which are G-proteincoupled receptors. We are presently asking if any of these known receptors might mediate ATP modulation of ASIC3.

\section{References}

1. Lewis T (1932). Pain in muscular ischemia. Archives of Internal Medicine, 49: 713-727.

2. Baker DG, Coleridge HM, Coleridge JC et al. (1980). Search for a cardiac nociceptor: stimulation by bradykinin of sympathetic afferent nerve endings in the heart of the cat. Journal of Physiology, 306: 519-536.

3. Tjen-A-Looi SC, Pan HL \& Longhurst JC (1998). Endogenous bradykinin activates ischaemically sensitive cardiac visceral afferents through kinin B2 receptors in cats. Journal of Physiology, 510: 633641.

4. James TN, Rossi L \& Hageman GR (1988). On the pathogenesis of angina pectoris and its silence. Transactions of the American Clinical and Climatological Association, 100: 81-99.

5. Gnecchi-Ruscone T, Montano N, Contini M et al. (1995). Adenosine activates cardiac sympathetic afferent fibers and potentiates the excitation induced by coronary occlusion. Journal of the Autonomic Nervous System, 53: 175-184.

6. Thames MD, Kinugawa T \& Dibner-Dunlap ME (1993). Reflex sympathoexcitation by cardiac sympathetic afferents during myocardial ischemia. Role of adenosine. Circulation, 87: 1698-1704.

7. Abe T, Morgan D, Sengupta JN et al. (1998). Attenuation of ischemia-induced activation of cardiac sympathetic afferents following brief myocardial ischemia in cats. Journal of the Autonomic Nervous System, 71: 28-36.

8. Pan HL \& Longhurst JC (1995). Lack of a role of adenosine in activation of ischemically sensitive cardiac sympathetic afferents. American Journal of Physiology, 269: H106-H113.

9. Huang MH, Horackova M, Negoescu RM et al. (1996). Polysensory response characteristics of dorsal root ganglion neurones that may serve sensory functions during myocardial ischaemia. Cardiovascular Research, 32: 503-515.

10. Huang HS, Pan HL, Stahl GL et al. (1995). Ischemia and reperfusion-sensitive cardiac sympathetic afferents: influence of $\mathrm{H}_{2} \mathrm{O}_{2}$ and hydroxyl radicals. American Journal of Physiology, 269: H888-H901.

11. Ginsburg R, Bristow MR, Kantrowitz N et al. (1981). Histamine provocation of clinical coronary artery spasm: implications concerning pathogenesis of variant angina pectoris. American Heart Journal, 102: 819-822.

12. Neill WA (1968). Myocardial hypoxia and anaerobic metabolism in coronary heart disease. American Journal of Cardiology, 22: 507515.

13. Pan HL, Longhurst JC, Eisenach JC et al. (1999). Role of protons in activation of cardiac sympathetic $\mathrm{C}$-fibre afferents during ischaemia in cats. Journal of Physiology, 518: 857-866.

14. Cobbe SM \& Poole-Wilson PA (1980). The time of onset and severity of acidosis in myocardial ischaemia. Journal of Molecular and Cellular Cardiology, 12: 745-760.

15. Reeh PW \& Steen KH (1996). Tissue acidosis in nociception and pain. Progress in Brain Research, 113: 143-151.

16. Krishtal OA \& Pidoplichko VI (1980). A receptor for protons in the 
nerve cell membrane. Neuroscience, 5: 2325-2327.

17. Krishtal OA \& Pidoplichko VI (1981). A "receptor" for protons in smal neurons of trigeminal ganglia: possible role in nociception. Neuroscience Letters, 24: 243-246.

18. Krishtal O (2003). The ASICs: signaling molecules? Modulators? Trends in Neurosciences, 26: 477-483.

19. Ueno S, Nakaye T \& Akaike N (1992). Proton-induced sodium current in freshly dissociated hypothalamic neurones of the rat. Journal of Physiology, 447: 309-327.

20. Akaike N \& Ueno S (1994). Proton-induced current in neuronal cells. Progress in Neurobiology, 43: 73-83.

21. Waldmann R, Champigny G, Bassilana F et al. (1997). A protongated cation channel involved in acid-sensing. Nature, 386: 173177.

22. Waldmann R \& Lazdunski M (1998). H(+)-gated cation channels: neuronal acid sensors in the NaC/DEG family of ion channels. Current Opinion in Neurobiology, 8: 418-424.
23. Benson CJ, Eckert SP \& McCleskey EW (1999). Acid-evoked currents in cardiac sensory neurons: A possible mediator of myocardial ischemic sensation. Circulation Research, 84: 921-928.

24. Immke DC \& McCleskey EW (2001). Lactate enhances the acidsensing $\mathrm{Na}^{+}$channel on ischemia-sensing neurons. Nature Neuroscience, 4: 869-870.

25. Sutherland SP, Benson CJ, Adelman JP et al. (2001). Acid-sensing ion channel 3 matches the acid-gated current in cardiac ischemiasensing neurons. Proceedings of the National Academy of Sciences, USA, 98: 711-716.

26. Cohen RD \& Woods HF (1983). Lactic acidosis revisited. Diabetes, 32: 181-191.

27. Forrester T (1972). An estimate of adenosine triphosphate release into the venous effluent from exercising human forearm muscle. Journal of Physiology, 224: 611-628.

28. Immke DC \& McCleskey EW (2003). Protons open acid-sensing ion channels by catalyzing relief of $\mathrm{Ca}^{2+}$ blockade. Neuron, 37: 75-84. 